\title{
Space charge characteristics of multi-layer oil-paper insulation under different DC voltages
}

\author{
Chao TANG ${ }^{1,2 *}, \mathrm{G} \mathrm{CHEN}^{2}, \mathrm{M} \mathrm{FU}^{3}$, Ruijin LIAO ${ }^{1}$ \\ 1 The State Key Laboratory of Power Transmission Equipment \& System Security and New Technology \\ Chongqing University, CHINA \\ 2 School of Electronics and Computer Science \\ University of Southampton, UK \\ 3 AVERA T\&D Technology Centre, Stafford, UK \\ *E-mail: tangchao_1981@163.com
}

\begin{abstract}
In this paper, space charge in oil-paper insulation system has been investigated using the pulsed electroacoustic (PEA) technique. A series of measurements were carried out when the insulation system was subjected to different applied voltages. Charge dynamics in the insulation system during the volts-on, volts-off and decay have been analyzed. It has been found that homocharge injection occurred both at the anode and the cathode. Positive charges are observed to accumulate in the layers, which indicate that the oil-paper layer interfaces act as a barrier for positive charges. The decay tests showed that after $30 \mathrm{~min}$, about a quarter of space charges remained in the sample, about $90 \%$ charges disappeared after 2 hours. Finally, total charge variation in these tests were analyzed.
\end{abstract}

Keywords: Space Charge, Oil-paper, DC Voltage, Multi-layer

\section{INTRODUCTION}

The oil-paper insulation has been used in power transformers, power cables and HVDC equipments for quite a long time because of its low cost and desirable physical and electrical properties. But they degrade under a combined stress of thermal, electrical, mechanical, and chemical stresses during routine operations, which will effect the lifetime of power equipments significantly. A lot of research works about the physical and electrical characteristics of oil-paper insulation have been conducted over the years. However, only a few studies about space charge in oil-paper insulation are reported.

Therefore, a better understanding of charge dynamics, in accordance with the specific variation of the charge distribution in multi layer insulation system becomes increasingly important and need a careful investigation. Thus, in this paper, based on the PEA technique, a series of measurements were carried out when the paper insulation system was subjected to different applied voltages. Charge dynamics in the insulation system during the volts-on and volts-off have been analyzed and the influence of temperature on charge dynamics discussed.

\section{EXPERIMENTS}

In this study, the measurements were carried out upon oil immersed insulation paper. The insulation paper which is made by Croylek Ltd, has a thickness of $\sim$ $60 \mu \mathrm{m}$ for single layer. The parameters of the paper meet the international standard IEC 554.2. The insulation oil used in this experiment was Nytro 10X transformer oil, which was provided by Nynas Oil Company.

Before PEA test, several pretreatment steps were carried out upon samples. Firstly, these new insulation paper were cut into round shape with a diameter of $\sim 2 \mathrm{~cm}$. Then the paper samples were kept in a vacuum oven at $393 \mathrm{~K}\left(120^{\circ} \mathrm{C}\right)$ for $20 \mathrm{~min}$, in order to make the moisture content less than $0.2 \%$. After that, the paper samples were impregnated by fresh degassed insulation oil in sealed oven. As thin paper was used, the sample can be fully impregnated after 1 day.

In practical application, oil-paper is usually used as multilayer insulation. Therefore, in this experiment, the PEA tests are performs on three layers oil-paper samples $(\sim 120 \mu \mathrm{m}$ after oil immersed and being pressed by electrodes, in Figure 1). The samples were stressed at three different DC voltage levels $(4 \mathrm{kV}, 6 \mathrm{kV}$, $8 \mathrm{kV}$ ) at the same temperature of $20^{\circ} \mathrm{C}$. Each time, an electric stressing time of 1 hour was tested. In addition, space charge evolution after the removal of the applied electric field was also measured.

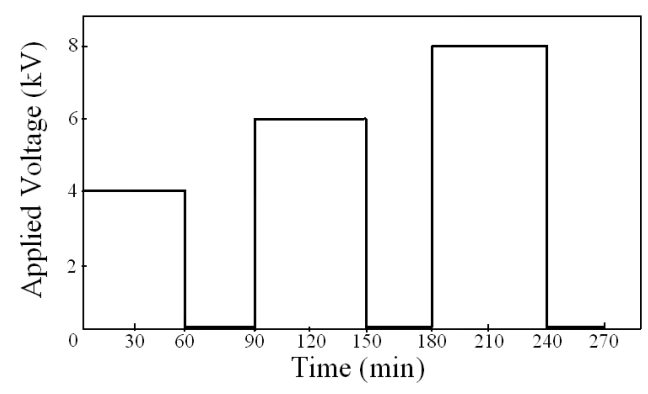

Figure 2. Voltage profile for space charge measurements

The space charge measurements were taken at various times during the periods of both volts-on and volts-off (short-circuit condition) using the PEA technique. In the PEA technique, acoustic pressure waves are generated due to the interaction of pulsed electric field and charge layer. Detection of acoustic pressure waves allows one to determine charge distribution across the sample. The principle of the PEA can be seen in many literatures $[1,2]$. The voltage profile in the present study is shown in Figure 2. 


\section{RESULTS AND DISCUSSION}

\subsection{Charge distribution with volts-on}

Obviously from the space charge distributions showed in Figure 4, the cathode peak is sharp and evident; in contrast, the anode peak is wide and flat, this is because of attenuation and scattering of acoustic wave caused by the oil-paper sample. Together with volts off figures, the positions of electrodes were confirmed and marked with black lines.

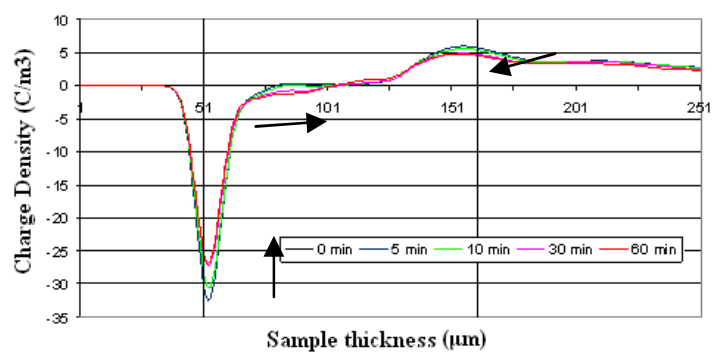

(a) $4 \mathrm{kV}$

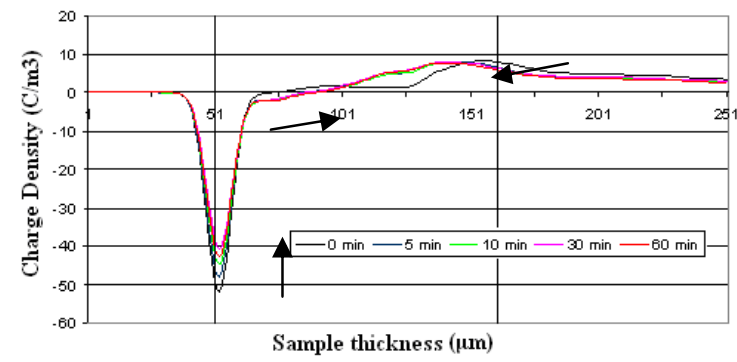

(b) $6 \mathrm{kV}$

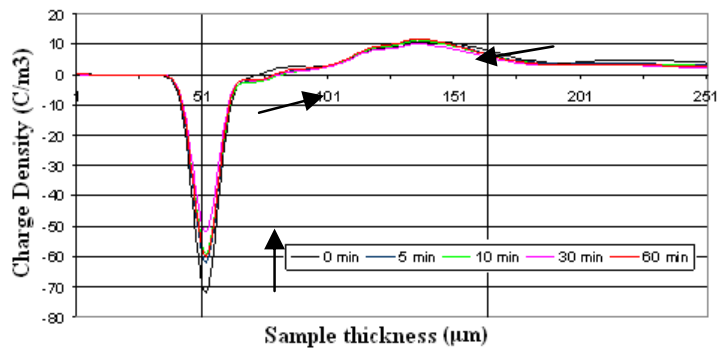

(c) $8 \mathrm{kV}$

Figure 4. Space charge dynamics at different dc electric fields (volts on)

As shown in Fig. 4 (a), (b) and (c), the peak value at electrodes increases with the applied voltage from $4 \mathrm{kV}$ to $8 \mathrm{kV}$. Under each applied voltage, because of homocharges injection at both electrodes, quantities of electric charges on both electrodes decreases as the stressing time increases. The positive charges accumulate in the vicinity of the anode, while the negative charges adjacent to the cathode. Charge injected quickly after voltage was switched on, an approximate equilibrium between positive and negative charge injection was observed after $30 \mathrm{~min}$. No big difference noticed after that time. However, it may take a relatively long period (e.g. up to 10,000 s), to reach the stable regime, i.e. the equilibrium between charge injection, extraction and transport [3].
The charge injection deepens into the bulk with the increase of the applied voltage. Under $4 \mathrm{kV}$, homocharges of each polarity enter no more than one layer. Under $6 \mathrm{kV}$, homocharges of each polarity enter more than one layer, it is possible that a small quantity neutralized in the middle layer. Under $8 \mathrm{kV}$, some homocharges reach the layer near to the opposite electrode.

Along with charge injection, electrode peaks offset towards the inner sample direction, especially the anode peak. There is less negative charge observed in the region adjacent to the cathode. There may be two reasons for this phenomenon. Firstly, compared to positive charge, the amount of negative charge injection didn't increase with the applied voltage, which indicates that negative charge may travel faster and with less blocked, and it reaches the anode quickly or is neutralized on the way. Secondly, the PEA technique only shows the net charge, the injected charge may be present together with the positive charge which is blocked at the interfaces.

Theoretically, in a PEA figure, the integral area of positive peaks is basically the same as that of negative peaks. However, because of the signal scattering in oil-paper sample and the contribution of pulse, the test results are some differences from that derived from theory.

\subsection{Charge distribution with volts-off}

Normally, Charges can be roughly classified as fast and slow charges $[4,5]$. 'Slow' charges are those which are essentially trapped in deep traps while 'fast' charges are those which escape from the traps very shortly after the dc voltage is removed. Charges trapped in the insulating material are often stable, i.e. slow charge. The volts-on measurement includes contributions from both fast and slow charges while the volts-off' measurement involves only slow charge.

After volts-on tests, slow charge distributions for three-layer samples under volts-off condition have been taken as shown in Figure 5. The charge distributions in the bulk hardly changes, while the induced charges form on both electrodes which confirmed the homocharge injection happened there under volts-on condition. What's more, the induced charge density increases gradually with the increase in the applied voltage (Fig. $5 \mathrm{a}, \mathrm{b}, \mathrm{c}$ ), from a maximum of $21 \mathrm{C} / \mathrm{m}^{3}(4 \mathrm{kV})$ to $26 \mathrm{C} / \mathrm{m}^{3}(6 \mathrm{kV})$ and then $33 \mathrm{C} / \mathrm{m}^{3}$ $(8 \mathrm{kV})$, which reflects the increase of charge injection.

Fig. 5 also shows that positive charges are dominant in the bulk after the removal of the applied voltage. It is noteworthy that only positive charges appears at the sample layer interfaces, which means the oil-paper layer interfaces act as a barrier for positive charges only. 


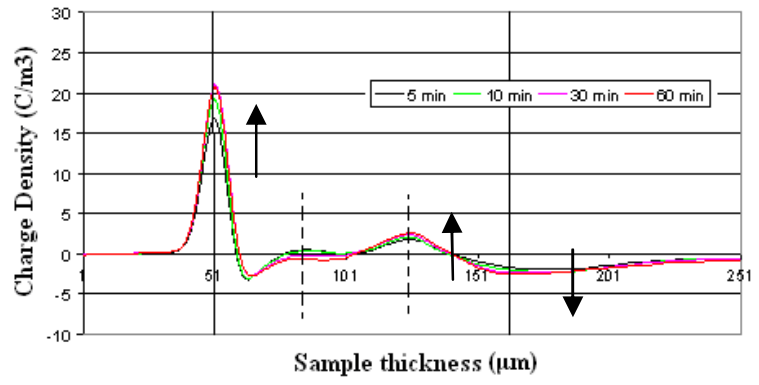

(a) $4 \mathrm{kV}$

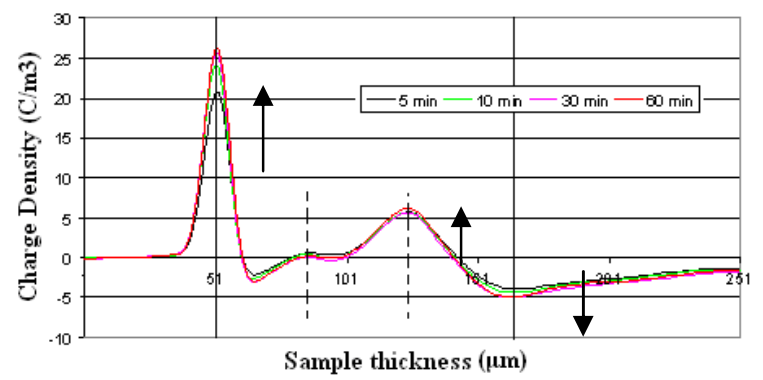

(b) $6 \mathrm{kV}$

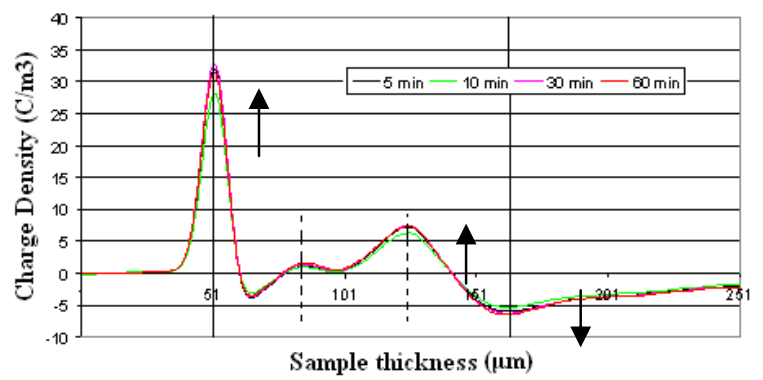

(c) $8 \mathrm{kV}$

Figure 5. Space charge dynamics at different dc electric fields (volts off)

\subsection{Charge distribution with decay}

After 1 hour dc stressing, space charge distributions after the removal of the applied voltage are shown in Figure 6. Compared to volts-on tests, charges are more easily injected than they are transported away from the electrodes. The charge decay speed becomes much more slowly with the time increase. After 30min, about a quarter of space charges remains in the sample. Further tests indicate that about $90 \%$ charges disappeared after 2 hours decay.

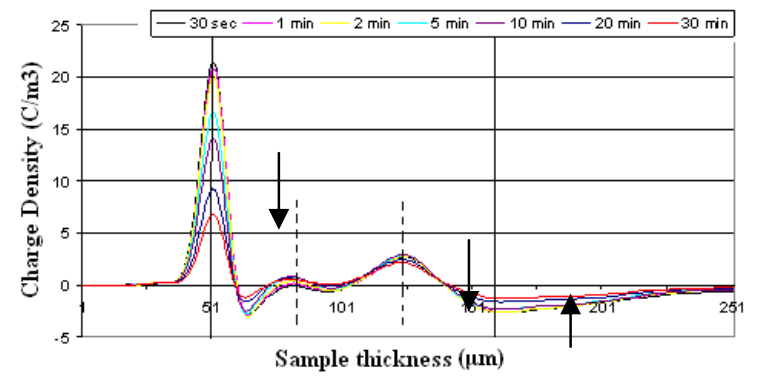

(a) $4 \mathrm{kV}$

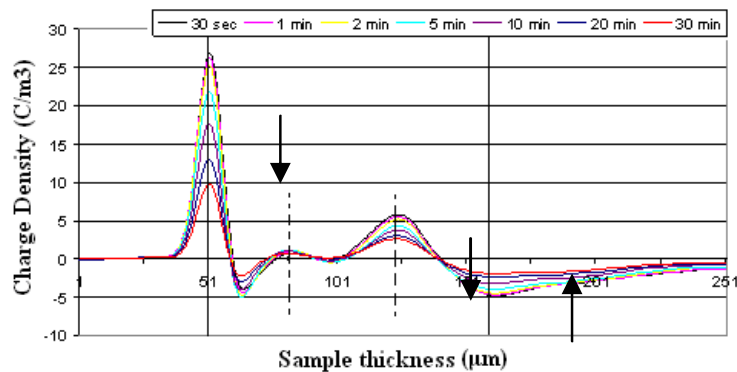

(b) $6 \mathrm{kV}$

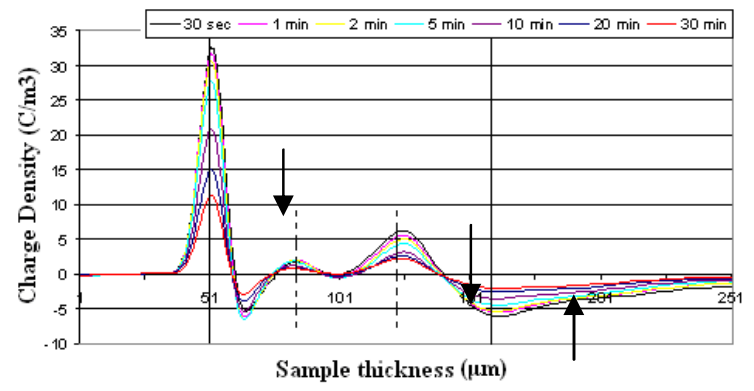

(c) $8 \mathrm{kV}$

Figure 6. Space charge decay after dc electric stressing

\section{4 total charge}

The total absolute amount of charge accumulated in the samples can be calculated based on the charge-density distribution given in Fig. 4-6.

$$
Q(T)=\int_{0}^{d}|\rho(x, t)| S d x
$$

Where $\rho(x, t)$ is the charge density, $S$ is the electrode area, and $d$ is the thickness of the sample. As we know, a trend of movement and combination exists between positive and negative charge. So the total charge is always changing during volts-on and volts-off tests. In this paper, the total charge at the time immediately after power on/off in the volts-on/volts-off test was calculated.

The relationship between the total amount of charge accumulated and the time under different applied voltages is shown in Fig. 7. Total charge in volts-on condition is the total injection charges including both fast moving charge and slow moving charge. At first, the higher the applied voltage is, the more charges injected. Secondly, a tendency of decline, then increase, finally to stable can be observed in Figure 7 (a). This can be explained as follows. At the very beginning, observable homocharge injected from both electrodes, and then fast moving charge moved quickly into the center of samples and neutralized, which made the total absolute amount of charge show a little decrease. After 30 min electrical stressing, it reached an approximate equilibrium between positive and negative charge. 


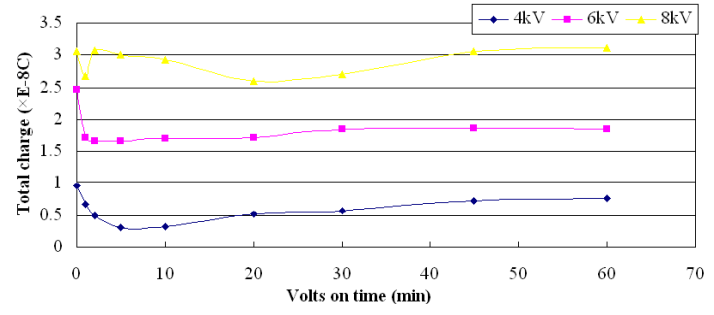

(a) Volts on

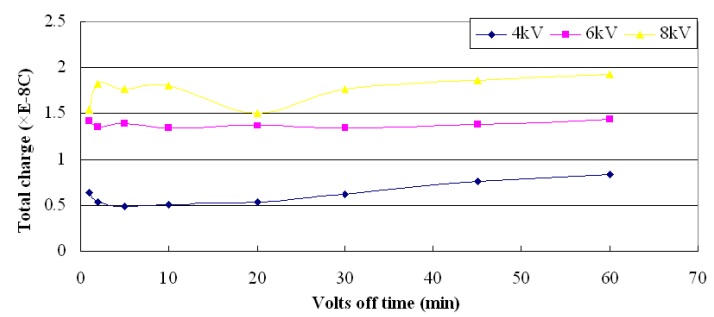

(b) Volts off

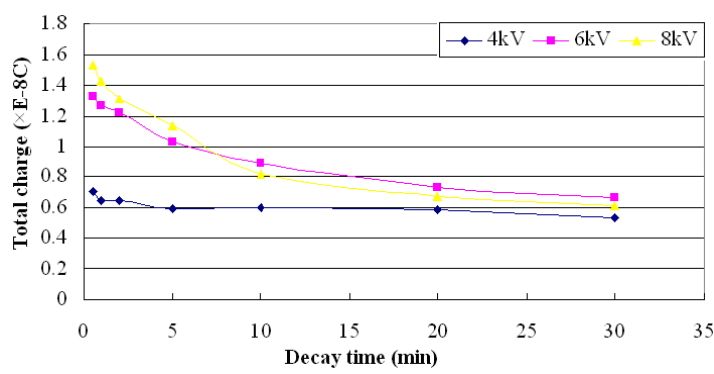

(c) Decay

Figure 7. Total charge under different applied voltages vs time.

Roughly, traps in electrical materials can be divided into two types, deep traps and shallow traps. When the charges moved into the deep traps, more energy is required for them to get out, which slow down the velocity of charge movement and finally formed slow moving charge. After power off, the volts-off tests were taken at once. The fast moving charges moved out immediately, only slow moving charges remain in the sample. In Figure 7 (b), the amount of slow moving charges in sample is stable except a little fluctuant at $8 \mathrm{kV}$. In addition, under different applied voltages, total charge at volts-off stabled in different horizontal. That may be explained as that, when increasing the applied voltage, the amount of injected charges in the material increases as well. As a result, more and more charges are trapped, the distortion of electric field becomes more serious, and the electrical performance gets worse.

It can be seen in Figure 7 (c), the higher the applied voltage is, the faster the charge decayed. As we know, at the early time when the power is off, more charges exist in higher voltage tested samples, which generated higher induction electrical field and made the charges moving faster and neutralized quickly.

\section{CONCLUSIONS}

In this paper, PEA measurements on multi-layer oil-impregnated insulation paper samples were presented. Space charge dynamics in volts-on, volts-off and decay are analyzed. The results are summarized as follows:

(1) Homocharge injection is observed both at the anode and the cathode, and the injection speed is very fast. The higher the applied voltage, the more the amount of injection charge and the deeper the charge injected into the sample layers.

(2) Positive charges are found to accumulate at the layer interfaces, which indicate that the oil-paper layer interfaces act as a barrier for positive charges. This will effect the distribution of electrical field and deteriorate the electrical behavior of oil-paper insulation.

(3) The decay tests showed that after 30min, about a quarter of space charges remained in the sample, about $90 \%$ charges disappeared after 2 hours.

In our opinion, both the electric and thermal stresses have significant impact to space charge behavior, further studies of space charge in oil-paper with variable applied voltages and temperatures are under performing which will be published later.

\section{ACKNOWLEDGEMENT}

One of the authors (Chao Tang) wishes to thank the 'Chinese Scholarship Council Funding for Joint Training $\mathrm{PhD}$ Student' for the financial support provided.

\section{REFERENCES}

[1] G. Chen, M. Fu, X. Z. Liu, L. S. Zhong. Ac aging and space-charge characteristics in low-density polyethylene polymeric insulation, Journal of Applied Physics, 2005, Vol. 97, No. 3, pp. 1-7.

[2] G. Chen, Y. Tanaka, T. Takada, L. Zhong. Effect of polyethylene interface on space charge formation. IEEE Transactions on Dielectrics and Electrical Insulation, 2005, Vol. 11, No. 1, pp. 113-121.

[3] R.C. Ciobanu, W. Pfeiffer, C. Schreiner. Charge packet evolution in paper-oil insulation and derived technological considerations. Proceedings of the 7th International Conference on Properties and Applications of Dielectric Materials, 2003, Vol. 2, pp. 695-698.

[4] G. Chen, Y. L. Chong, M. Fu. Calibration of the pulsed electroacoustic technique in the presence of trapped charge. Measurement Science and Technology, 2006, Vol. 17, No. 7, pp. 1974-1980.

[5] Mingli Fu, George Chen, Len A. Dissado, John C. Fothergill. Influence of thermal treatment and residues on space charge accumulation in XLPE for DC power cable application, IEEE Transactions on Dielectrics and Electrical Insulation, 2007, Vol. 14, No. 1, pp. 53-64. 